\title{
La procalcitonina in Terapia Intensiva: un grande futuro alle spalle?
}

\section{Procalcitonin in Intensive Care: a great future behind?}

\author{
Romolo M. Dorizzi ${ }^{1} \cdot$ Piersandro Sette $^{2}$
}

Ricevuto: 7 gennaio 2016 / Accettato: 25 gennaio 2016 / Pubblicato online: 25 febbraio 2016

(C) Società Italiana di Patologia Clinica e Medicina di Laboratorio 2016

Riassunto Il confine tra informazione e promozione di un farmaco o di un esame, attività sicuramente utili sia per l'industria sia per il professionista sanitario, clinico e laboratorista, e "marketing" con la sola finalità di sommergere il possibile utilizzatore con dati con poche o nulle informazioni (o addirittura fuorvianti) non è sempre netto. Come esempio di un esame a rischio di essere interessato da questo fenomeno viene affrontato il caso della procalcitonina (PCT). Si tratta di un esame commercializzato da circa vent'anni che si è diffuso rapidamente, anche perché è oggi disponibile sulle piattaforme presenti praticamente in tutti i Laboratori clinici di qualunque dimensione. È richiesto soprattutto dalle Unità Operative di Terapia Intensiva ed è stato inserito in algoritmi per guidare la somministrazione degli antibiotici. Tuttavia, negli ultimi anni è stato segnalato da più parti che, nonostante una teorica base fisio-patologica non vi sono prove univoche che l'inserimento della determinazione della PCT in algoritmi per la gestione del paziente settico sia efficace. Anche il NICE ha recentemente concluso che l'esame è promettente, ma non vi sono ancora prove sufficienti per raccomandarne l'uso in Terapia Intensiva per guidare la decisione di sospendere la somministrazione di antibiotici in pazienti con sepsi. Il Laboratorio rimane un attore cruciale per la definizione, l'applicazione e l'aggiornamento di linee guida che prevedono l'esecuzione di esami di laboratorio e dovrà partecipare nel lavoro futuro, che anche secondo il NICE deve continuare, per confermare o smentire l'efficacia della PCT. In questo ambito gli strumenti classici della Evidence Based Laboratory Medicine, come il nomogramma

R.M. Dorizzi

romolo.dorizzi@auslromagna.it

1 U.O.C. Patologia Clinica, AUSL della Romagna, Piazzale della Liberazione 60, 47522 Pievesestina di Cesena, FC, Italia

2 U.O.S. Gestione Gruppo Operatorio, U.O.C. Direzione Medica, Ospedale "G. Fracastoro", San Bonifacio, VR, Italia di Fagan, possono aiutare clinico e laboratorista a stimare il valore diagnostico e quindi l'efficacia della PCT.

Parole chiave Procalcitonina - Terapia Intensiva . Algoritmi · NICE $\cdot$ Linee guida $\cdot$ Nomogramma di Fagan

Summary The line between information and promotion of a drug and a test, activity definitely useful both for the industry and for the health professional, clinician and laboratorian, and "marketing" for the sole purpose of overwhelming the prospective user with data with little or no (or even misleading) information is not always clear. As an example of a test at risk of being affected by this phenomenon we discuss procalcitonin (PCT), a test marketed by some twenty years that has spread rapidly, partly because it is available on all the platforms present in virtually clinical laboratories of any size. It is requested especially by the Intensive Care Units and it has been included in algorithms to guide the administration of antibiotics. However, in recent years several reports concluded that, despite a theoretical physio-pathological base, there is no unambiguous evidence that the inclusion of PCT in algorithms is effective in the management of septic patients. Also NICE has recently concluded that the test is promising but there is still insufficient evidence to recommend its use in intensive care to guide the decision to stop the use of antibiotics in patients with sepsis. The laboratory remains a crucial player for the development, implementation and updating of guidelines including laboratory tests and will have to participate in the future work, that even according to NICE must continue, to confirm or refute the PCT effectiveness. In this context, the traditional tools of Evidence Based Laboratory Medicine as the Fagan nomogram can be very useful for the clinician and the laboratorian to assess the diagnostic value and therefore the effectiveness of the PCT. 
Keywords Procalcitonin - Intensive Care - Algorithms . NICE $\cdot$ Guidelines $\cdot$ Fagan nomogram

\section{Introduzione}

Il ruolo dell'industria in medicina e, particolarmente in Medicina di Laboratorio, è un argomento complesso con numerosi aspetti. È sempre più importante che l'industria contribuisca a tenere informato e aggiornato il professionista circa gli esami e i dispositivi che sono messi a punto e commercializzati. Tuttavia, negli anni recenti le strategie di marketing delle aziende di diagnostici sono diventate sempre più vicine a quelle impiegate dall'industria farmaceutica da mezzo secolo. Quanto ha dichiarato nel 1976 Henry Gadsen, in procinto di lasciare la guida della Merck, alla rivista Fortune sintetizza in modo impareggiabile quell' approccio "Il nostro sogno è produrre farmaci per i sani perché questo ci consentirebbe di venderli a tutti [1]". La pressione dell'industria ha ormai medicalizzato eventi naturali quali la gravidanza, l'invecchiamento, la menopausa. Da più parti è stato raccomandato che in sanità l'industria fornisca informazioni rilevanti, valide e provate e che i professionisti partecipino nella valutazione delle informazioni in modo critico [2].

Uno degli autori che ha studiato di più questo problema è John Ioannidis, che sostiene che una parte non trascurabile, se non la maggioranza, di quanto è riportato in letteratura (trials clinici, articoli di ricerca, studi epidemiologici) non è confermata o è, addirittura, smentita dalla letteratura successiva. Secondo Ioannidis [3] il rischio che risultati di uno studio non siano confermati è tanto più grande quanto più (1) gli studi compiuti in un settore sono piccoli, (2) l'effetto investigato è piccolo, (3) il numero delle relazioni investigate è grande, (4) la flessibilità del disegno, delle definizioni, degli outcome e dei metodi impiegati è maggiore, (5) i conflitti di interesse economico e di altro tipo e i pregiudizi scientifici sono maggiori, (6) l'interesse che in quel momento suscita quell'ambito di ricerca è maggiore e, di conseguenza, (7) i gruppi di ricercatori che vi operano sono numerosi. Ioannidis è un autore molto seguito e il lavoro apparso su Chance e Plose One è stato citato nei 10 anni successivi alla pubblicazione oltre 3000 volte e affronta un tema che ha risvolti che toccano anche la Medicina di Laboratorio, in cui si verificano casi in cui interessi più o meno evidenti del ricercatore o del professionista associato a quello di una o più aziende offuscano le vere dimensioni e solidità della qualità e dell'utilità di un strumento o di un reagente. Ne consegue che sono oggetto di promozioni molto pesanti e si verifica che sono introdotti strumenti e reagenti di sicuro interesse, ma per i quali non è stato ancora dimostrato in modo univoco il ruolo, non tanto nella ricerca, ma nella diagnostica routinaria.

Il caso della procalcitonina (PCT) è significativo a questo riguardo e rappresenta uno di quelli più rilevanti nell'ultimo decennio. È stato uno dei temi di maggiore interesse nella nostra attività professionale che ha portato alla preparazione di articoli che contano oggi circa 450 citazioni [4-6] e rimane oggi, dopo 15 anni, un esame che seguiamo attentamente dal versante laboratoristico (RMD) e clinico (PS).

In questa sede può essere utile esaminare come è stata gestita la richiesta dell'attivazione dell'esecuzione della PCT in una U.O. di Terapia Intensiva della Romagna. Alla fine del 2012 è stata inviata la richiesta di introduzione di tale esame con la seguente motivazione: Utilizzo della determinazione dei valori di PCT nei pazienti con infezione sospetta o certa ricoverati in Terapia Intensiva, al fine di ottimizzare l'impiego della terapia antibiotica in termini di appropriatezza-efficacia-durata, ridurne i consumi, contenere il costo economico e la pressione ambientale negativa da questi esercitata con conseguente selezione di germi Multi-Drug-Resistent. La richiesta era corredata da una Rassegna della Cochrane Collaboration [7] ed è stata sollecitata da altri Direttori delle UU.OO. di Terapia Intensiva in genere accompagnata dalla stessa Cochrane Collaboration. Nella motivazione di uno di questi solleciti si leggeva: "Se il problema è economico (argomento assolutamente condivisibile) l'introduzione della PCT potrebbe riservarci sorprese positive ottimizzando l'appropriatezza, la durata e l'efficacia di impiego dell'antibioticoterapia. In Italia sono ormai rimasti pochi gli ospedali di un certo spessore che non forniscono la possibilità di dosare la PCT".

La Cochrane Collaboration merita di essere esaminata per verificare da una parte qual è la capacità/disponibilità/ volontà dei colleghi clinici di esaminare in modo critico la letteratura e dall'altra l'attualità dell'articolo di Ioannidis.

La rassegna comprende 14 trials con 4221 partecipanti; cinque di questi sono stati compiuti in Terapia Intensiva e sono brevemente riassunti di seguito.

- Bouadma et al [8] hanno pubblicato un trial randomizzato multicentrico realizzato in nove UU.OO. francesi di Terapia Intensiva che ha coinvolto un gruppo di 307 pazienti in cui la decisione di iniziare e di sospendere la terapia antibiotica era presa sulla base della concentrazione della PCT e un gruppo di 314 pazienti in cui la decisione era presa sulla base della valutazione clinica. L'algoritmo utilizzato prevedeva la sospensione dell'antibiotico (AB) quando la concentrazione di PCT si abbassava dell' $80 \%$ rispetto alla concentrazione picco, ovvero raggiungeva un valore inferiore a $0,5 \mu \mathrm{g} / \mathrm{L}$. La PCT era misurata 7 giorni a settimana e il risultato era comunicato ai medici entro 2 ore dal prelievo. Cochrane Collaboration ha analizzato solo i 394 pazienti con diagnosi di infezione acuta respiratoria. Un bias segnalato dagli autori della Cochrane Collaboration è che in più della metà dei casi (53\%) l'algoritmo NON è stato seguito dagli autori. Lo studio è stato finanziato da Assistance Publique-Hopitaux 
de Paris e Brahms (Thermofisher) ha fornito tutti i reagenti (e la strumentazione per i centri che non ne erano dotati) e alcuni autori sono stati consulenti dell'azienda o hanno ricevuto onorari.

- Hochreiter et al [9] hanno pubblicato nel 2010 un trial randomizzato svolto in una U.O. di Terapia Intensiva tedesca comprendente 110 pazienti chirurgici trattati con antibiotici (un gruppo di 57 pazienti in cui la decisione di sospendere la somministrazione di antibiotici era presa sulla base della concentrazione della PCT e un gruppo di 53 pazienti in cui la terapia era somministrata per 8 giorni). L'algoritmo utilizzato prevedeva la sospensione della terapia antibiotica quando i segni clinici e i sintomi di infezione "miglioravano" e la concentrazione di PCT scendeva sotto $1 \mu \mathrm{g} / \mathrm{L}$ o restava più alta ma diminuiva entro 3 giorni del $25-35 \%$ rispetto al valore iniziale. La PCT era misurata 7 giorni a settimana su base "routinaria", ma non è indicato entro quanto tempo dal prelievo il risultato era comunicato ai medici. Cochrane collaboration ha analizzato solo i 43 pazienti con diagnosi di infezione acuta respiratoria. Un bias segnalato dagli autori della review è che non è indicato in quanti casi l'algoritmo NON è stato seguito dagli autori. Un autore è stato consulente dell'azienda e ha ricevuto onorari.

- Nobre et al [10] hanno pubblicato nel 2009 un trial randomizzato realizzato in una U.O. di Terapia Intensiva svizzera comprendente 79 pazienti trattati con antibiotici (un gruppo di 39 pazienti in cui la decisione di sospendere gli antibiotici era presa sulla base della concentrazione della PCT e un gruppo di 40 pazienti in cui la terapia era somministrata secondo le modalità "standard"). Lo studio è stato finanziato da Brahms (Thermofisher). L'algoritmo utilizzato prevedeva di misurare la PCT 7 giorni a settimana entro 1 ora dal prelievo e il risultato era comunicato ai medici entro 3 ore dal prelievo. I pazienti con PCT al basale $\geq 1 \mu \mathrm{g} / \mathrm{L}$ erano rivalutati il giorno 5 . I clinici erano incoraggiati a sospendere la terapia antibiotica quando la concentrazione di PCT:

- scendeva più del $90 \%$ della concentrazione di base ovvero

- raggiungeva un valore inferiore a $0,25 \mu \mathrm{g} / \mathrm{L}$.

I pazienti con PCT al basale $<1 \mu \mathrm{g} / \mathrm{L}$ erano rivalutati al giorno 3. I clinici erano incoraggiati a sospendere la terapia antibiotica quando la concentrazione di PCT era inferiore a $0,1 \mu \mathrm{g} / \mathrm{L}$ e un'attenta valutazione clinica "escludeva un'infezione severa". Cochrane Collaboration ha analizzato solo i 25 pazienti con diagnosi di infezione respiratoria. Un bias segnalato dagli autori della review è che l'algoritmo NON è stato seguito dagli autori nel $19 \%$ dei casi. Alcuni autori hanno ricevuto finanziamenti o hanno ricevuto onorari da Brahms (Thermofisher).

- Schroeder et al [11] hanno pubblicato nel 2009 un trial randomizzato in una U.O. di Terapia Intensiva tedesca comprendente 27 pazienti trattati con antibiotici (un gruppo di 14 pazienti in cui la decisione di sospendere la somministrazione di antibiotici era presa sulla base della concentrazione della PCT e un gruppo di 13 pazienti di controllo in cui la terapia era interrotta sulla base dei segni clinici e di “empiric rules"). L'algoritmo utilizzato prevedeva la determinazione della PCT tutti i giorni. La terapia antibiotica era interrotta quando i segni clinici e i sintomi di infezione "miglioravano" e la concentrazione di PCT scendeva sotto $1 \mu \mathrm{g} / \mathrm{L}$ o restava più alta ma diminuiva del $25-35 \%$ dal valore iniziale entro 3 giorni consecutivi. La PCT era misurata 7 giorni a settimana in condizioni "routinarie", ma non è indicato entro quante ore dal prelievo il risultato era comunicato ai medici. Un bias segnalato dagli autori della rassegna è che non è indicato in quanti pazienti l'algoritmo NON è stato seguito dagli autori. Un autore ha ricevuto onorari da Brahms (Thermofisher).

- Stolz et al [12] hanno pubblicato nel 2009 un trial multicentrico randomizzato in 7 UU.OO. di Terapia Intensiva in Europa e USA comprendente 101 pazienti trattati con antibiotici. L'algoritmo utilizzato prevedeva di iniziare la terapia antibiotica secondo il giudizio clinico. Dopo 72 ore era comunicato il valore di PCT.

- Il clinico era "incoraggiato fortemente" a sospendere la terapia antibiotica quando la concentrazione di PCT era $<0,25 \mu \mathrm{g} / \mathrm{L}$.

- Il clinico era "incoraggiato" a sospendere la terapia antibiotica quando la concentrazione era tra $0,25 \mathrm{e}$ $0,5 \mu \mathrm{g} / \mathrm{L} \mathrm{o} \geq 80 \%$ rispetto al giorno 0 ovvero una concentrazione $>0,5 \mu \mathrm{g} / \mathrm{L}$.

- Il clinico era "scoraggiato" a sospendere la terapia antibiotica quando la concentrazione di PCT era $\geq 0,5 \mu \mathrm{g} / \mathrm{L}$ o era diminuita $<80 \%$ rispetto al giorno 0 .

- Il clinico era "fortemente scoraggiato" a sospendere la terapia antibiotica quando la concentrazione di PCT era $>1 \mu \mathrm{g} / \mathrm{L}$.

La PCT era misurata 10 giorni in routine e la risposta era inviata entro 1 ora dal prelievo. Un bias segnalato dagli autori è che non è indicato in quanti casi l'algoritmo NON è stato seguito dagli autori. Lo studio è stato finanziato da organizzazioni pubbliche e private e Brahms (Thermofisher) ha fornito reagenti e logistica per lo studio.

\section{Un commento alla Cochrane Collaboration}

Ma la Cochrane Collaboration arriva davvero a risultati solidi e trasferibili "chiavi in mano" (applicabili immediatamente) alle UU.OO. di Terapia Intensiva e di conseguenza al Laboratorio? Consideriamo alcuni aspetti:

(1) Nove dei 18 autori della Cochrane collaboration sono autori anche degli articoli compresi nella rassegna. In 
particolare, tutti gli autori dei 5 articoli che si occupano dell'impiego della PCT in Terapia Intensiva prima considerati sono stati i revisori di se stessi nella rassegna.

(2) Gli studi che riguardano la Terapia Intensiva comprendono un numero limitato di pazienti.

(3) La diminuzione dei giorni di somministrazione di antibiotici nei pazienti in cui la terapia è stata guidata della determinazione della PCT è rispettivamente di:

- 3 giorni (media) per Bouadma et al (da 13,3 a 10,3 giorni);

- 2 giorni (media) per Hochreiter et al (da 7,9 a 5,9 giorni);

- 3 giorni (mediana) per Nobre da 9,5 giorni (3-34) a 6 giorni (2-33) [la differenza della media non è indicata];

- 1,7 giorni per Schroeder (da 8,3 a 6,6 giorni);

- 3,5 giorni (media) per Stolz da 13 giorni (2-21) a 9,5 giorni (1,5-17).

(4) Non può sfuggire quanto segnalato da Bouadma et al [8] (a slightly higher number of patients in the procalcitonin group than in the control group died between days 29 and 60, potentially questioning the safety of a procalcitonin-guided strategy in the intensive care unit, hospital, and rate of relapse e questo è uno degli aspetti dell'articolo maggiormente discussi [13-17].

Si tratta di un dato che ha una concretezza da considerare anche a fronte della riportata minore esposizione agli antibiotici dei pazienti inseriti nel gruppo gestiti con il monitoraggio della PCT.

(5) Molto importanti dal punto di vista operativo sono la diversità degli algoritmi con cui è interpretato il valore di PCT proposti e l'elevata percentuale di casi in cui il clinico non ha seguito l'algoritmo ma la "clinica" (peraltro indicata in qualche caso in modo molto generico). In sostanza il Laboratorio che introduce l'esame nel proprio repertorio non ha oggi indicazioni precise e standardizzate su modi e tempistica con cui introdurre l'esame.

(6) A ben guardare, comunque, le conclusioni degli autori della Cochrane collaboration sono ben distanti dall'essere conclusive e dal considerare la PCT un esame con un ruolo definito e consolidato e quindi "maturo" per l'inserimento nella pratica clinica. Recita, infatti: "Further high-quality research is needed to confirm the safety of this approach for non-European countries and patients in intensive care. Moreover, future studies should also establish cost-effectiveness by considering countryspecific costs of procalcitonin measurement and potential savings in consumption of antibiotics and other healthcare resources, as well as secondary cost savings due to lower risk of side effects and reduced antimicrobial resistance".
(7) D'altra parte non possono essere altre le conclusioni se gli articoli che sono stati meta-analizzati in ambito intensivistico concludono, a loro volta:

- Bouadma: “...A procalcitonin-guided strategy could reduce antibiotic selective pressure with potential benefits in the era of multiresistance".

- Hochreiter: “...PCT-controlled antibiotic therapy must still be tested in heterogenous groups of patients, particularly for safety".

- Nobre: “...A multicenter trial enrolling a large number of patients with severe sepsis and septic shock to test our PCT guidance protocol and its effect on ICU length of stay is desirable to validate our data".

- Schroeder: "Monitoring of PCT ... may contribute to an optimized antibiotic regimen with beneficial effects on microbial resistances and costs in intensive care medicine".

- Stolz: “..., we achieved only modest gains in antibiotic use for VAP. This may be due to additional diffculties in the interpretation of procalcitonin results in such ill patients, potentially related to higher proportion of previous systemic inflammatory response syndromes, multiorgan failure, previous infection and/or surgery, all known to raise the procalcitonin levels".

Con grande rammarico, dato l'interesse verso l'esame che gli autori di questo editoriale hanno avuto "dal principio", nell'AUSL della Romagna 2013 si è concluso che non vi erano prove che l'outcome dei pazienti ricoverati in Terapia Intensiva fosse migliorato dall'introduzione della PCT.

In effetti la pressione delle aziende che commercializzano l'esame è continuata nel nostro Paese come nel resto del mondo, tanto che nel luglio 2014 il NICE ha iniziato una valutazione con un obiettivo molto semplice "The purpose of this assessment is to evaluate the clinical and cost effectiveness of using procalcitonin testing with standard clinical practice to guide antibiotic treatment in the following populations:

- Adults and children with confirmed or highly suspected sepsis in intensive care settings.

- Adults and children with suspected bacterial infection presenting to the emergency department".

L'autorevolezza raggiunta dal NICE ha alimentato le attese per le sue conclusioni che avrebbero sicuramente influenzato l'atteggiamento di clinici e laboratoristi non solo nel Regno Unito. Sono state considerate non solo le fonti classiche come Medline ed Embase, ma anche quelle irrituali come NHS economic Evaluation Database, Health Economic Evaluation Database, EconLit e Research Papers in Economics. Le raccomandazioni del NICE sono state rese pubbliche nell'ottobre 2015 [18]. 
NICE passa in rassegna 18 studi (8 svolti in terapia intensiva e 10 nei dipartimenti di Emergenza) che in parte erano stati compresi anche nella Cochrane Collaboration del 2012. Dal punto di vista metodologico rileva che nessuno studio è stato compiuto nel Regno Unito (la maggior parte in Svizzera, 3 in Cina e 1 in Brasile) e che 3 studi presentavano un rischio alto di bias, 1 basso, mentre per tutti gli altri non era possibile definire il rischio di bias [19].

Le conclusioni della NICE Guidance sono piuttosto tranchant "The procalcitonin tests (ADVIA Centaur BRAHMS PCT assay, BRAHMS PCT Sensitive Kryptor assay, Elecsys BRAHMS PCT assay, LIAISON BRAHMS PCT assay and VIDAS BRAHMS PCT assay) show promise but there is currently insufficient evidence to recommend their routine adoption in the NHS. Further research on procalcitonin tests is recommended for guiding decisions to stop antibiotic treatment in people with confirmed or highly suspected sepsis in the intensive care unit or start and stop antibiotic treatment in people with suspected bacterial infection presenting to the emergency department. Centres currently using procalcitonin tests to guide these decisions are encouraged to participate in research and data collection".

Come da prassi consolidata le conclusioni sono sintetizzate anche nella sezione che il sito del NICE dedica alle informazioni per il pubblico "There was not enough evidence to recommend that these tests are used in the NHS. But NICE has recommended further research and data collection to show the impact of adding procalcitonin testing to standard clinical practice in the NHS".

La saga della PCT non è sicuramente conclusa (fortunatamente), ma il documento NICE fissa un punto fermo per le prossime puntate. Poco potranno aggiungere singoli studi che comprendono una dozzina di pazienti. Laboratorio, industria e clinici possono sicuramente continuare a lavorare in questo ambito per raccogliere ulteriori prove, ma partendo da questa base. L'obiettivo del NICE era molto semplice "The NICE Diagnostics Assessment Programme will assess the clinical and cost-effectiveness of the BRAHMS PCT Sensitive Kryptor assay (and other alternative technologies identified during scoping) in order to make recommendations on their use in the NHS". Uno degli aspetti attraenti di questo sforzo era quindi di coniugare l'aspetto scientifico a quello pratico, vale a dire che il NICE voleva rispondere a chi si chiedeva e chiedeva se il Sistema Sanitario Nazionale del Regno Unito poteva/doveva dotarsi di un esame non solo efficace ma anche cost-effective per ottimizzare il consumo di antibiotici in Terapia Intensiva (e nel Dipartimento di Emergenza). Ha pertanto costituito un Comitato di professionisti che comprendeva microbiologi, intensivisti, pediatri, laboratoristi e infermieri e ha richiesto a tutti i produttori dei reagenti per l'esame (Thermofisher, Roche, Siemens, Diasorin, Biomerieux) di inviare qualunque materiale ritenessero potesse dimostrare il ruolo e l'importanza dell'esame. Il NICE ha considerato tutto il materiale inviato e lo ha incorporato nella raccomandazione finale o ha dato le motivazione sulla base delle quali non l'ha fatto [20].

In sostanza il NICE conclude che la PCT rimane un esame di ricerca che non è ancora "maturo" per essere inserito nel Sistema Sanitario Nazionale del Regno Unito; la riduzione del consumo di antibiotici che alcuni autori hanno segnalato nei pazienti inseriti in un algoritmo basato sulla determinazione della PCT è legata più all'impiego di un protocollo clinico definito piuttosto che alla misurazione della PCT.

Il NICE conviene che la ricerca debba andare avanti e, mentre segnala che Shebabi et al [che avevano usato un cutoff di $0,1 \mu \mathrm{g} / \mathrm{L}]$ non avevano riportato una riduzione significativa nell'impiego degli antibiotici nel braccio in cui era misurata la PCT [21], lamenta che lo studio randomizzato SAPS (Stop Antibiotics on guidance of Procalcitonin Stu$d y$ ), anche se completato, non aveva ancora prodotto alcun articolo [22].

Mentre colpisce che le conclusioni di un documento di un organismo così autorevole come il NICE abbiano avuto così poca risonanza, non si può fare altro che augurarsi che la posizione di organismi senza conflitti di interessi possa essere nota almeno quanto gli altri numerosi articoli che sono capillarmente portati alla conoscenza dei laboratoristi e dei clinici.

Ulteriori elementi relativi allo studio possono ricavati dal Rapporto HTA che dovrebbe diffonderne la conoscenza e l'applicabilità [23].

Altri studi recenti meritevoli di attenzione sono quello di Annane et al, che non hanno confermato che un algoritmo basato sulla PCT possa influenzare l'uso di antibiotici [24], e quello di Brechot et al, che riporta come l'algoritmo basato sulla PCT non porti alla riduzione del consumo di antibiotici e solo il $46 \%$ dei pazienti con bassa PCT non riceva antibiotici. La riluttanza del medico a basare l'inizio della terapia con antibiotici sulla concentrazione di PCT dei pazienti critici è attribuita dalla bassa accuratezza del biomarcatore di rilevare l'infezione con batteri [25].

Prkno et al lamentano le sostanziali differenze tra i diversi algoritmi proposti, che dovranno essere risolte negli studi futuri "Reliable cut-off values of PCT levels to guide therapeutic decisions need to be defined in future studies, since treatment algorithms varied substantially between the studies included in our systematic review ... Most importantly, treatment algorithms differ substantially and have to be clarified in future studies" [26]. In quella che è diventata una costante degli articoli sulla PCT la rassegna sistematica di Prkno et al ha ricevuto numerosi commenti. Particolarmente interessante quello di Salluh et al [27] "Despite their important contribution, most trials evaluating PCT-guided antibiotic therapy present several limitations that preclude their safe extrapolation to the clinical decision-making process. Namely, the high rate of patient exclusion (reaching 
$>80 \%$ in the Svoboda and colleagues trial) and the high rate of algorithm overruling (reaching $>50 \%$ in the PRORATA trial) disregard the impact of renal failure as well as renal replacement therapy on PCT levels, and above all the heterogeneity of duration of antibiotic therapy in the controls".

Elegante è anche la rassegna sistematica di Wacker et al [28], che conferma l'interesse più speculativo che operativo della PCT. Le conclusioni sembrano scontate "Procalcitonin is a helpful marker for diagnosis of sepsis in critically ill patients. However, it cannot be recommended as the single definitive test for sepsis diagnosis, but rather it must be interpreted in context with information from careful medical history, physical examination, and when feasible, microbiological assessment. Moreover, continuing re-evaluation during the course of disease is advisable", ma sono corredate da un vivido approccio EBM semplice ma efficace. Ribadita la difficoltà che la grande differenza di cut-off impiegati (mediana di 1,1 $\mu \mathrm{g} / \mathrm{L}$ ) introduce nell'impiego di algoritmi in clinica gli autori trovano una sensibilità e una specificità combinata dei 22 studi meta-analizzati rispettivamente del $77 \%$ e del $79 \%$ con una LR+ di 3,67 e una LR- di 0,29 ben distanti da quelle ottimali. C'è da chiedersi quale clinico nella nebbia della diagnosi si soffermerà a impiegare un nomogramma di Fagan o, comunque un approccio bayesiano, ma a quali conclusioni arriverebbe lui o il paziente o l'amministratore di un' azienda USL se considerassero quanto emerge dalle Figura 1 e 2 . In un paziente in cui la probabilità pre-test di sepsi, sulla base della clinica, è del $10 \%$ un risultato di PCT positivo la porta al $29 \%$ e uno negativo la porta al $3 \%$, ma in un paziente in cui il quadro è più incerto, con una probabilità pre-test di sepsi del $50 \%$ un risultato di PCT positivo la porta al $79 \%$ e uno negativo la porta al $22 \%$. Percentuale sufficiente per sospendere la terapia antibiotica?

\section{PCT e Drotrecogin}

Dopo 4 anni dal ritiro dal commercio della "molecola miracolo", la proteina $\mathrm{C}$ attivata ricombinante (Drotrecogin alfa attivato: Xigris), rimane ancora valida la raccomandazione di esaminare sempre attentamente la letteratura disponibile prima di introdurre una nuova procedura terapeutica $o$ diagnostica.

Il caso Xigris è stato ampiamente discusso su numerosi giornali tra cui New England Journal of Medicine [29] e JAMA [30]. Wikipedia [31] sintetizza gli eventi (in una voce presente solo in inglese, tedesco, polacco, portoghese, serbo, serbo-croato e svedese) iniziati nel 2001 quando Eli Lilly ha commercializzato Xigris, un farmaco approvato per la terapia della sepsi. Tutto era cominciato con il fascicolo dell'8 marzo 2001 del New England Journal of Medicine che conteneva i risultati dello studio PROWESS, che an-

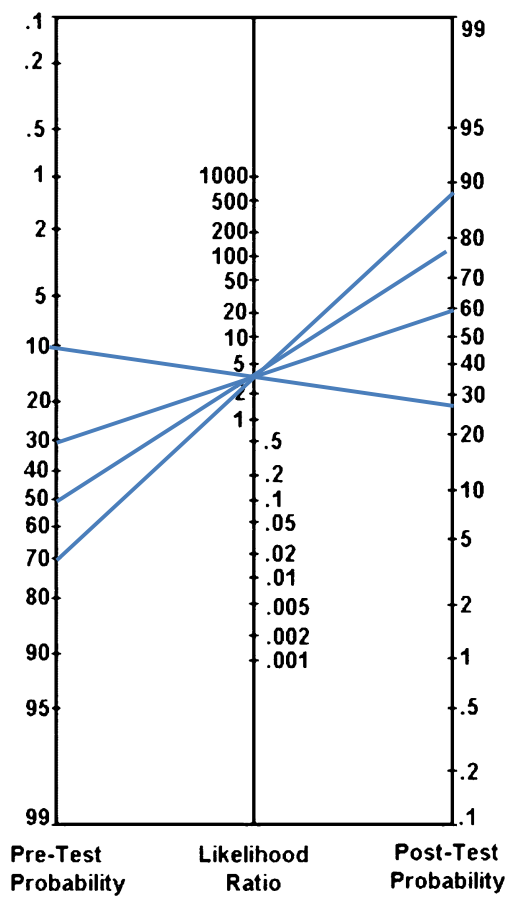

Fig. 1 Probabilità post-test calcolata a una Probabilità Pre-test del $10 \%, 30 \%, 50 \%$ e $70 \%$ e il Likelihood Ratio Positivo ricavato da Ref. [28]

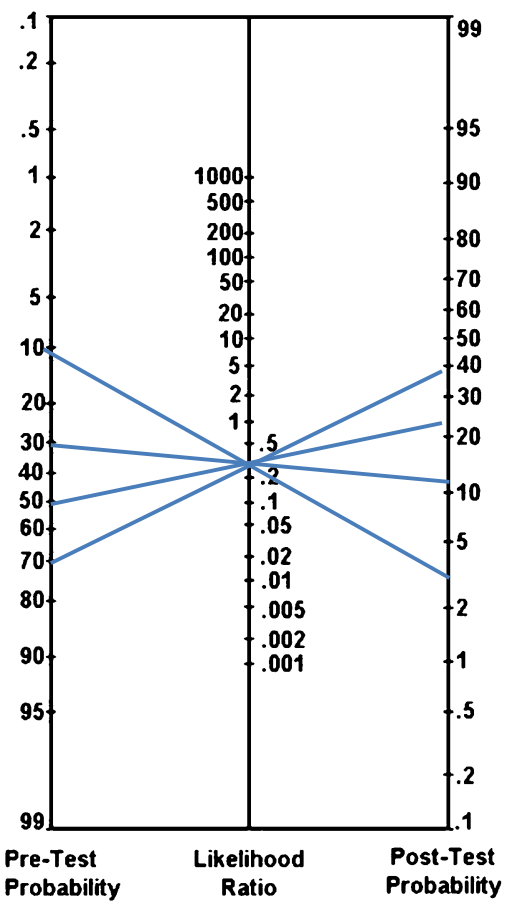

Fig. 2 Probabilità post-test calcolata a una Probabilità Pre-test del $10 \%, 30 \%, 50 \%$ e $70 \%$ e il Likelihood Ratio Negativo ricavato da Ref. [28]

nunciava una straordinaria novità nel trattamento della sepsi, la drotrecogin alfa, una forma ricombinante attivata della 
proteina $\mathrm{C}$, l'anticoagulante endogeno [32]. L'editoriale che accompagnava l'articolo affermava "At last, however, there has been progress in finding an effective new therapy for sepsis [33]".

La promozione del farmaco è stata intensa e il Values, Ethics, and Rationing in Critical Care Task Force è stato finanziato con 1,8 milioni di dollari con lo scopo dichiarato di affrontare gli aspetti etici del "razionamento" in medicina intensiva. La Food and Drug Administration (FDA) aveva rallentato negli Stati Uniti l'approvazione di drotrecogin con l'indicazione di ridurre la mortalità nella sepsi severa (score APACHE di almeno 25) dal momento che allo Xigris era stato associato sin dall'inizio un aumento di emorragie severe [34]. La reale efficacia del farmaco continuò a essere discussa negli Stati Uniti e in Europa fino a quando, il 25 ottobre 2011, Eli Lilly annunciò il ritiro volontario a livello mondiale dello Xigris. Lo stesso giorno, solo qualche ora più tardi, furono resi pubblici i risultati del trial PROWESS-SHOCK, che non confermavano i risultati del trial PROWESS e non ne dimostravano l'efficacia [35]. Erano, pertanto, confermate le conclusioni della Cochrane Collaboration dell'aprile 2011 "This updated review found no evidence suggesting that APC should be used for treating patients with severe sepsis or septic shock. Additionally, APC is associated with a higher risk of bleeding. Unless additional RCTs provide evidence of a treatment effect, policy-makers, clinicians and academics should not promote the use of APC [36]".

Il ruolo del Laboratorio nel tradurre nel mondo reale linee guida cliniche che hanno generalmente una bassa considerazione per gli aspetti "laboratoristici" è stato recentemente discusso su questo giornale [37]. Cappelletti ha auspicato che SIPMeL, come Società e come Gruppi di Studio, svolga un ruolo sempre più attivo all'interno del percorso complesso e cumulativo rappresentato dalla Medicina Traslazionale e dia indicazioni, suggerimenti e raccomandazioni che rendano più veloce l'implementazione di approcci up-to-date ed evidence based del singolo professionista o, meglio, dell'equipe di laboratorio a tutte le fasi del total testing process, per rispondere adeguatamente al quesito clinico esplicito o implicito in ogni richiesta di esame di laboratorio [37].

Negli ultimi vent'anni abbiamo numerosi casi di raccomandazioni abbandonate pochi anni dopo essere state proposte da esperti come "best practices" ("preoperative $\beta$ blocker, tight glucose control in the intensive care unit, activated protein $C$ for sepsis, hormone replacement therapy for women after menopause" [38]).

Clinici, laboratoristi e amministratori devono basare la loro pratica su basi solide, devono rimanere sempre aggiornati e pronti a modificarla rapidamente quando nuovi dati e nuove "prove" diventano disponibili.

Sicuramente il caso della PCT è diverso da quello dello Xigris, ma le conclusioni del NICE suggeriscono l'opportunità, in attesa che per lo meno si chiuda lo studio SAPS, che:
- i laboratori che non eseguono la PCT non la attivino;

- quelli che la eseguono ma non hanno dati di reale riduzione del consumo di antibiotici ne sospendano l'esecuzione;

- quelli che negli anni passati hanno raccolto dati del genere ne continuino l'impiego ma contribuiscano al progresso della professione rendendo pubblici i loro dati.

In conclusione, di fronte alla Diagnostic Guidance del NICE del 7 ottobre 2015 si può concludere quanto è stato scritto nel 2012 su drotrecogin “The investigators' findings provide a sad chapter in the noble quest for a truly effective adjunct for the treatment of septic shock. This setback should inspire a redoubling of efforts to seek new approaches to treatment that are based on a more crystalline view of the biology of sepsis" [39]. Senza dimenticare un altro editoriale di qualche anno prima del New England Journal of Medicine "The challenges involved in producing first rate guidelines and performance standards are only exacerbated by the intrusion of marketing strategies masquerading as evidence based medicine" [29].

\section{Conflitti di interesse Nessuno.}

Studi condotti su esseri umani e animali L'articolo non contiene alcuno studio eseguito su esseri umani e su animali da parte dell'autore.

\section{Bibliografia}

1. Moynihan R, Cassels A (2005) Selling sickness. Nation Books, New York

2. Cambronero-Saiz B (2007) Pharmaceutical advertising: from prospectus to marketing. J Epidemiol Community Health 61:366

3. Ioannidis JP (2005) Why most published research findings are false. PLoS Med 2:e124

4. Luzzani A, Polati E, Dorizzi R et al (2003) Comparison of procalcitonin and C-reactive protein as markers of sepsis. Crit Care Med 31:1737-1741

5. Dorizzi RM, Polati E, Sette P et al (2006) Procalcitonin in the diagnosis of inflammation in intensive care units. Clin Biochem 39:1138-1143

6. Santuz P, Soffiati M, Dorizzi RM et al (2008) Procalcitonin for the diagnosis of early-onset neonatal sepsis: a multilevel probabilistic approach. Clin Biochem 41:1150-1155

7. Schuetz P, Müller B, Christ-Crain M et al (2012) Procalcitonin to initiate or discontinue antibiotics in acute respiratory tract infections. Cochrane Database Syst Rev 9:CD007498

8. Bouadma L, Luyt CE, Tubach F et al (2010) Use of procalcitonin to reduce patients' exposure to antibiotics in intensive care units (PRORATA trial): a multicentre randomised controlled trial. Lancet 375:463-474

9. Hochreiter M, Köhler T, Schweiger AM et al (2009) Procalcitonin to guide duration of antibiotic therapy in intensive care patients: a randomized prospective controlled trial. Crit Care 13:R83

10. Nobre V, Harbarth S, Graf JD et al (2008) Use of procalcitonin to shorten antibiotic treatment duration in septic patients: a randomized trial. Am J Respir Crit Care Med 177:498-505

11. Schroeder S, Hochreiter M, Koehler T et al (2009) Procalcitonin (PCT)-guided algorithm reduces length of antibiotic treatment in surgical intensive care patients with severe sepsis: re- 
sults of a prospective randomized study. Langenbecks Arch Surg 394:221-226

12. Stolz D, Smyrnios N, Eggimann P et al (2009) Procalcitonin for reduced antibiotic exposure in ventilator-associated pneumonia: a randomised study. Eur Respir J 34:1364-1375

13. Kollef $\mathrm{MH}$ (2010) Will procalcitonin reduce antibiotic use in intensive care? Lancet 375:435-436

14. Mokart D, Leone M (2010) Procalcitonin in intensive care units: the PRORATA trial. Lancet 375:1605

15. Tarnow-Mordi W, Gebski V (2010) Procalcitonin in intensive care units: the PRORATA trial. Lancet 375:1605

16. Gibot S (2010) Procalcitonin in intensive care units: the PRORATA trial. Lancet 375:1605-1606

17. Landman GW, Kleefstra N (2010) Procalcitonin in intensive care units: the PRORATA trial. Lancet 375:1606

18. NICE (2015) Procalcitonin testing for diagnosing and monitoring sepsis (ADVIA Centaur BRAHMS PCT assay, BRAHMS PCT Sensitive Kryptor assay, Elecsys BRAHMS PCT assay, LIAISON BRAHMS PCT assay and VIDAS BRAHMS PCT assay). https://www.nice.org.uk/guidance/dg18 (Accesso 3 gennaio 2016)

19. NICE (2015). https://www.nice.org.uk/guidance/DG18/ documents/diagnosis-and-monitoring-of-sepsis-procalcitonintesting-final-protocol2 (Accesso 3 gennaio 2016)

20. NICE (2015). https://www.nice.org.uk/guidance/DG18/ documents/consultation-comments-and-response (Accesso 3 gennaio 2016)

21. Shehabi Y, Sterba M, Garrett PM et al (2014) Procalcitonin algorithm in critically ill adults with undifferentiated infection or suspected sepsis. a randomized controlled trial. Am J Respir Crit Care Med 190:1102-1110

22. Assink-de Jong E, de Lange DW, van Oers JA et al (2013) Stop antibiotics on guidance of procalcitonin study (SAPS): a randomised prospective multicenter investigator-initiated trial to analyse whether daily measurements of procalcitonin versus a standardof-care approach can safely shorten antibiotic duration in intensive care unit patients—calculated sample size: 1816 patients. BMC Infect Dis 13:178

23. Westwood M, Ramaekers B, Whiting P et al (2015) Procalcitonin testing to guide antibiotic therapy for the treatment of sepsis in intensive care settings and for suspected bacterial infection in emergency department settings: a systematic review and cost-effectiveness analysis. Health Technol Assess 19:1-236

24. Annane D, Maxime V, Faller JP et al (2013) Procalcitonin levels to guide antibiotic therapy in adults with non-microbiologically proven apparent severe sepsis: a randomised controlled trial. BMJ Open 3:e02186

25. Bréchot N, Hékimian G, Chastre J et al (2015) Procalcitonin to guide antibiotic therapy in the ICU. Int J Antimicrob Agents 46(Suppl 1):S19-S24

26. Prkno A, Wacker C, Brunkhorst FM et al (2013) Procalcitoninguided therapy in intensive care unit patients with severe sepsis and septic shock - a systematic review and meta-analysis. Crit Care 17:R291

27. Salluh JI, Nobre V, Povoa P (2014) Using procalcitonin to guide antimicrobial duration in sepsis: asking the same questions will not bring different answers. Crit Care 18:142

28. Wacker C, Prkno A, Brunkhorst FM et al (2013) Procalcitonin as a diagnostic marker for sepsis: a systematic review and metaanalysis. Lancet Infect Dis 13:426-435

29. Eichacker PQ, Natanson C, Danner RL (2006) Surviving sepsispractice guidelines, marketing campaigns, and Eli Lilly. N Engl J Med 355:1640-1642

30. Angus DC (2011) The search for effective therapy for sepsis: back to the drawing board? JAMA 306:2614-2615

31. Wikipedia. https://en.wikipedia.org/wiki/Drotrecogin_alfa (Accesso 3 gennaio 2016)

32. Bernard GR, Vincent JL, Laterre PF et al (2001) Efficacy and safety of recombinant human activated protein $\mathrm{C}$ for severe sepsis. N Engl J Med 344:699-709

33. Matthay MA (2001) Severe sepsis - a new treatment with both anticoagulant and antiinflammatory properties. N Engl J Med 344:759-762

34. Warren HS, Suffredini AF, Eichacker PQ et al (2002) Risks and benefits of activated protein $\mathrm{C}$ treatment for severe sepsis. N Engl J Med 347:1027-1030

35. Ranieri VM, Thompson BT, Barie PS et al (2012) Drotrecogin alfa (activated) in adults with septic shock. N Engl J Med 366:20552064

36. Martí-Carvajal AJ, Solà I, Lathyris D et al (2011) Human recombinant activated protein $\mathrm{C}$ for severe sepsis. Cochrane Database Syst Rev 4:CD004388

37. Cappelletti P (2015) Linee guida e SIPMeL. Riv Ital Med Lab 11:185-190

38. Neuman MD, Bosk CL, Fleisher LA (2014) Learning from mistakes in clinical practice guidelines: the case of perioperative $\beta$-blockade. BMJ Qual Saf 23:957-964

39. Wenzel RP, Edmond MB (2012) Septic shock—evaluating another failed treatment. N Engl J Med 366:2122-2124 